\title{
Electrostatic Interactions Shape Molecular Organization and Electronic Structure of Organic Semiconductor Blends
}

Gabriele D’Avino, ${ }^{* \dagger}$ Steffen Duhm, ${ }^{\ddagger}$ Raffaele Guido Della Valle, Georg Heimel, ${ }^{\S}$ Martin Oehzelt, $"$ Satoshi Kera, ${ }^{\perp}$ Nobuo Ueno,\# David Beljonne, ${ }^{@}$ and Ingo Salzmann ${ }^{*, \Delta}$

$\dagger$ †nstitut Néel CNRS \& Grenoble Alpes University, Grenoble, France

$\ddagger$ Institute of Functional Nano \& Soft Materials (FUNSOM), Jiangsu Key Laboratory for

Carbon-Based Functional Materials \& Devices and Joint International Research Laboratory of

Carbon-Based Functional Materials and Devices, Soochow University, Suzhou, People's

Republic of China

๑Dipartimento di Chimica Industriale Toso Montanari, Università di Bologna/INSTM-UdR

Bologna, Italy

§Institut für Physik, Humboldt-Universität zu Berlin, Germany

||Helmholtz-Zentrum Berlin für Materialien und Energie GmbH, Berlin, Germany

$\perp$ Institute for Molecular Science, Okazaki, Japan

\#Graduate School of Advanced Integration Science, Chiba University, Japan

@Laboratory for the Chemistry of Novel Materials, University of Mons, Belgium

$\triangle$ Department of Physics, Department of Chemistry \& Biochemistry, Centre for Research in Molecular Modeling (CERMM), Centre for NanoScience Research (CeNSR), Concordia University, Montreal, Canada

E-mail: gabriele.davino@neel.cnrs.fr; ingo.salzmann@concordia.ca 


\begin{abstract}
Halogenation of conjugated molecules represents a powerful approach to tune the electronic structure of molecular thin-films through inductive effects and long-range intermolecular electrostatic interactions. The mixing of halogenated molecules with their pristine counterparts has recently proven successful in altering the blend's energy levels to adjust the open-circuit voltage of organic solar cells by the mixing ratio. Here, we show that the prevailing rationale for this effect is not equally valid for different molecular orientations. We provide a comprehensive experimental and theoretical analysis of the prototypical blend formed by pentacene and perfluoropentacene to relate structure with electronic properties. We find a mixed-stack structural motif in standing and lying orientation depending on the substrate nature. In standing orientation, the ionization potential lies in between the values of the pure components, in line with the established picture of averaged molecular quadrupole moments. For the lying orientation, however, we experimentally observe an ionization potential lower than both pristine values, which seems at odds with this simple rationale. Electrostatic simulations based on the knowledge of the atomistic structure of the films capture the complex experimental scenario for both orientations. In particular, the ultra-low ionization potential of films formed by lying molecules is identified as a signature of the monolayer structure, where quadrupolar interactions are responsible for a difference of ca. $0.4 \mathrm{eV}$ in the highest occupied molecular orbital energy as compared to thicker films with the same molecular orientation.
\end{abstract}




\section{Introduction}

Functional structures based on $\pi$-conjugated molecules and polymers show high potential in cost-effective, lightweight and flexible opto-electronic devices, such as organic light-emitting diodes, solar cells, transistors, and sensors. ${ }^{1,2}$ However, translating promising properties of isolated molecules into functional materials is far from trivial, as their opto-electronic properties in thin films strongly depend on the underlying molecular arrangement. In this context, polycyclic aromatic hydrocarbons, their halogenated derivatives and heterostructures thereof, provide a particularly versatile platform for understanding the intimate relationship between molecular and materials properties. This is best reflected in the large number of studies that have explored the structural, optical, and electronic characteristics of such systems both experimentally and at various levels of theory during the last decade. ${ }^{1,3-10}$ The deep interest in these systems is rooted in the unique opportunities that halogenation offers for tuning excitonic and charge-transport energy levels over a wide range. The functionalization of the $\pi$-conjugated molecular cores with fluorine permits tuning the electronic properties through the inductive effect exerted by these electron-withdrawing substituents ${ }^{11,12}$ and via long-range electrostatic interactions in the solid state. ${ }^{3,13-17}$ The latter effect results from intermolecular interactions governed both by molecular properties (the electrical multipole moments) and supramolecular organization. There, it has been demonstrated that the presence of intramolecular polar bonds equally impacts the ionization potential (IP) and the electron affinity in ordered films of small conjugated molecules ${ }^{3,18-21}$ and polymers. ${ }^{22}$

For the prototypical material pair pentacene (PEN) and perfluoropentacene (PFP) it has been shown that the IP of pure films strongly depends on the molecular orientation, ${ }^{23,24}$ which can be readily modified by using different substrates. Films of uprightstanding molecules are typically found on dielectric substrates like $\mathrm{SiO}_{\mathrm{x}}$, while an inplane orientation of the long molecular axes prevails on metallic substrates, highly oriented pyrolytic graphite (HOPG), and graphene. ${ }^{25-27}$ For these extreme cases, a large IP 
(a)

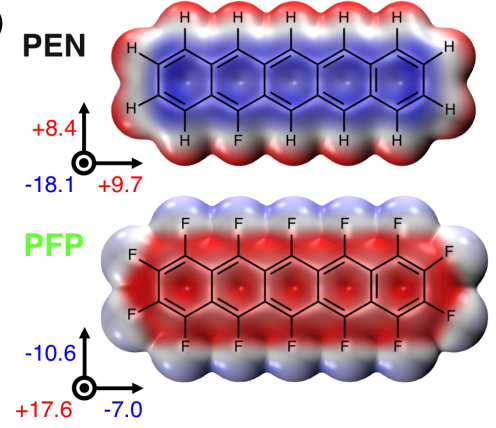

(b)

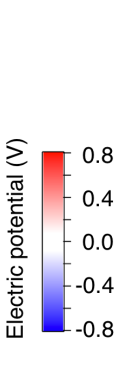

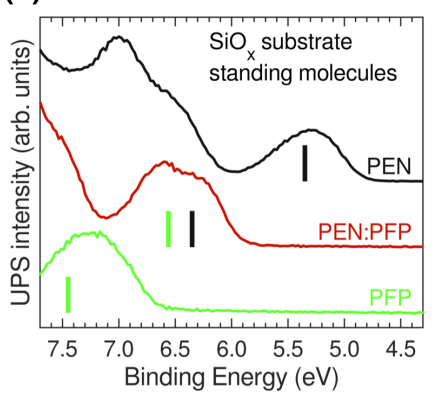

(c)

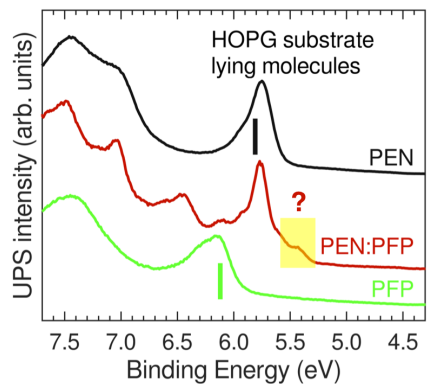

Figure 1: (a) Chemical structure and electrostatic properties (electrostatic potential color map and principal components of the molecular quadrupole $Q$ in Debye. $\AA$ ) of PEN and PFP molecules. (b) Experimental UPS data (given in binding energy w.r.t. the vacuum level, i.e., IP) for PEN, PFP, and 1:1 co-deposited PEN:PFP films of on $\mathrm{SiO}_{\mathrm{x}}$ comprising standing molecules (taken from Ref. 18). (c) Experimental UPS data for films of lying molecules on HOPG, including monolayers of pristine PEN and PFP ( $4 \AA$ nominal thickness), and 1:1 co-deposited PEN:PFP mixed films ( $8 \AA$ nominal thickness). In contrast to the case of $\mathrm{SiO}_{\mathrm{x}}$, the IP of the mixed film does not lie between the values of the pristine materials, but is lower than both. black and green vertical bars in $(b, c)$ mark our calculation results for the IP of PEN and PFP, respectively.

difference has been reported between PEN films formed by lying (IP = 5.5 eV, HOPG) and standing ( $\left(\mathrm{IP}=4.8 \mathrm{eV}, \mathrm{SiO}_{\mathrm{x}}\right)$ molecules, as derived from the low binding energy (BE) onset of the corresponding ultraviolet photoelectron spectroscpoy (UPS) data (black curves in Fig. 1b-c). This trend is reversed for PFP, where films of lying molecules $(\mathrm{IP}=5.9 \mathrm{eV})$ have a lower IP compared to films in standing orientation $(\mathrm{IP}=6.7 \mathrm{eV}$, green curves in Fig. 1b-c). This phenomenon has been rationalized by electrostatic effects brought about by the respective molecular quadrupoles of PEN and PFP (see Fig. 1a), whose principal components are reversed with respect to each other owing to the opposite polarity of the C-H and C-F bonds. Therefore, differences in IP between the molecules in lying and standing orientation are, in fact, understood by the opposite orientation of the molecular quadrupole components.

As to be expected, heterostructures of such dissimilar compounds stand on a significantly higher level of complexity with a wide range of achievable morphologies and microstructures, where the resulting structural and electronic properties can subtly de- 
pend on the preparation conditions. For instance, layered heterostructures of PEN and PFP obtained by sequential vacuum deposition on $\mathrm{SiO}_{\mathrm{x}}$ have been shown to grow in the same (standing) orientation as the individual compounds on the bare substrate, therefore retaining the IP values of the individual films. ${ }^{24}$ Conversely, vacuum co-deposition of PEN and PFP (on $\mathrm{SiO}_{\mathrm{x}}$ ) yields films intermixed on the molecular scale. There, the growth of a crystalline mixed PEN:PFP phase with 1:1 stoichiometry on $\mathrm{SiO}_{\mathrm{x}}$ has been discussed in numerous studies, as deduced from specular (XRD) and grazing incidence X-ray diffraction (GIXRD), ${ }^{18,28}$ electron diffraction, ${ }^{29}$ optical absorption ${ }^{18,28,30,31}$ and vibrational spectroscopy. ${ }^{32}$ This mixed phase has been found to be remarkably stable ${ }^{33}$ and has been shown to prevail also in the excess of one of the two components. ${ }^{18,28,29}$ However, no full structure solution of the PEN:PFP mixed crystal structure has been reported to date, as both compounds are essentially insoluble inhibiting the preparation of macroscopic samples suitable for single-crystal X-ray diffraction.

For the electronic structure of such blends, one could naively expect a simple superposition of the UPS signals associated with the pure films for non-interacting molecules. However, the IP of mixed films lies, instead, between the values of the pristine materials, as illustrated in Fig. $1 \mathrm{~b}$ by UPS data taken from Ref. 18 for PEN, PFP, and 1:1 mixed films thereof. For practical applications, this finding therefore allows tuning the IP of functional films in organic electronic devices by varying the mixing ratio. This concept has recently been exploited by Schwarze et al. for related materials in organic solar cells, and allowed to continuously tune the effective donor energy levels and the open-circuit voltage of the cell with the mixing ratio. ${ }^{15,34}$ The initial interpretation of this observation was framed in terms of an effective average of the electrostatic potential exerted by PEN and PFP molecules featuring opposite quadrupole moments. ${ }^{18}$ Later, a more detailed, atomistic modeling of the UPS data was performed assuming a mixed crystal structure based on the known herringbone arrangement found for the pure compounds. ${ }^{35}$ While these calculations indeed predicted IP values in the correct energy range, a quantitative 
comparison to the experiment was hindered by the lack of knowledge of the underlying crystal structure.

In the present work, we aim to challenge these simple perceptions of the mechanism underlying IP tuning in molecular heterostructures. The need for a more detailed view is best demonstrated by UPS data of a 1:1 mixed PEN:PFP film formed by lying molecules (red curve in Fig. 1c), which shows an IP value as low as $5.4 \mathrm{eV}$. In clear contrast to the same blend in standing orientation (red curve in Fig. 1b), the IP is now lower than both individual values of the pure compounds. To understand this surprising observation that appears to be at odds with a simple electrostatic explanation based on opposite molecular quadrupoles, we provide a comprehensive, atomistic picture for the structural and electronic properties of this prototypical organic semiconductor blend, thereby shedding light on the subtle interplay between structure and electrostatics in this system. Reliable simulations for this system, however, require the precise knowledge of the underlying crystal structure, which we provide via full structure solutions of PEN:PFP blends from GIXRD experiments supported by crystal structure modeling, both for the lying and standing orientations. This allows for the sound application of an atomistic, classical, electrostatic scheme encompassing charge-multipole interactions and image-charge effects to assess the energy landscape of charge carriers in PEN:PFP as a function of the film thickness. As a result, the experimentally observed energy-level positions emerge naturally, thereby providing the theoretical framework necessary for reliable electronic-structure engineering through blending dissimilar organic semiconductors on the molecular scale.

\section{Experimental Section}

Thin Film Growth and UPS: Pure and mixed films of PEN and PFP for UPS were prepared by vacuum (co-)deposition on clean HOPG surfaces using resistively heated quartz crucibles with deposition rates of about $0.25 \AA / \mathrm{min}$, as controlled by a quartz crystal mi- 
crobalance. HOPG (ZYA grade) was cleaved in air just before loading into the preparation chamber and cleaned by in situ heating at $420{ }^{\circ} \mathrm{C}$ for $11 \mathrm{~h}$. UPS experiments were performed using a custom built ultra-high vacuum apparatus (base pressure $<$ $4 \times 10^{-10}$ mbar) equipped with a HeI UV-light-source and a hemispherical electron energy analyzer (Scienta R3000). The angle between the incident beam and the sample was fixed to $65^{\circ}$. The spectra were measured at photoelectron emission angles $(\theta)$ of $0^{\circ}$ (normal emission) and $45^{\circ}$ with an acceptance angle of $\pm 10^{\circ}$; a sketch of the specific experimental geometry can be found in Ref. 36. The energy resolution was set to $80 \mathrm{meV}$. The error of UPS BE values is estimated to be $\pm 0.05 \mathrm{eV}$. For the measurement of the secondary electron cutoff (SECO) the sample was biased at $-3.00 \mathrm{~V}$. All preparation steps and measurements were performed at room temperature $(295 \mathrm{~K})$.

$X R D$ and GIXRD: For structural analysis, pure and mixed films of PEN and PFP were grown on freshly cleaved HOPG (ZYA quality) by physical vapour (co-)deposition in the high vacuum (final nominal film thickness $30 \mathrm{~nm}$; base pressure $<5 \times 10^{-8} \mathrm{mbar}$; deposition rate per compound ca. $1 \AA / \mathrm{min}$ ). The films were characterized at beamline W1 at the synchrotron radiation facility DORIS (HASYLAB, Hamburg). GIXRD experiments were performed immediately after XRD (same alignment) using a goniometer in pseudo $2+2$ geometry and a one-dimensional detector (MYTHEN, Dectris); the use of a primary and secondary slit system (evacuated flight tube with entrance and exit slits behind the sample) allowed for recording high resolution data (which is not possible in a 2D detector setup) for the precise determination of peak positions; the primary beam energy was $10.5 \mathrm{keV}$. GIXRD experiments were performed using incident angles of the primary beam relative to the HOPG substrate of $0.15^{\circ}$. Reciprocal space maps were recorded by keeping the sample fixed and by performing a series of detector scans along the in-plane scattering angle at differently fixed out-of-plane scattering angle. The vertical mounting of the detector allows the simultaneous measurement of $3.5^{\circ}$ in out-of-plane direction. The experimental diffraction pattern was transformed to reciprocal space and compared to the 
calculated peaks emerging from the structure solutions using the custom-made software PyGID. ${ }^{37}$

Crystal Structure Modeling: Intermolecular interactions in the solid state have been modeled by a classical force field including an atom-atom potential plus a Coulombic contribution described by atomic charges from electrostatic potential fitting (ESP scheme). ${ }^{38}$ Molecules have been considered as rigid bodies, with geometries determined by gasphase optimization at the B3LYP/6-31G(d) level of theory using the Gaussian suite. ${ }^{39}$ For the atom-atom model, we have considered several commonly used force fields, tested by computing the crystallographic structures of several pure PEN and PFP forms, ${ }^{25,40-42}$ and finally selected a Lennard-Jones atom-atom potential with the OPLS-AA parameters. ${ }^{43,44}$ The latter yields root mean square deviations between experimental and calculated lattice parameters around $0.05 \AA$ for the cell axes and $0.4^{\circ}$ for the cell angles.

Electronic Structure Calculations: Band structure and intermolecular charge-transfer integrals have been computed at the DFT level employing the PBE0 functional ${ }^{45}$ and the 6-31G* basis set. Band structure calculations have been performed for the PEN:PFP crystal structure determined on the HOPG substrate with the all-electron CRYSTAL14 code. ${ }^{46}$ Charge transfer integrals were evaluated with the dimer projection method ${ }^{47}$ on the basis of DFT calculations performed with the ORCA 4.0 code. ${ }^{48}$

The IPs of thin films have been calculated using the well-established perturbative framework for localized charge carriers ${ }^{14}$ as

$$
\mathrm{IP}=\mathrm{IP}_{\text {gas }}+\Delta
$$

where $\mathrm{IP}_{\text {gas }}$ is the the IP of the isolated molecule (taken from experiments: $6.60 \mathrm{eV}$ for PEN; ${ }^{49,50} 7.50 \mathrm{eV}$ for $\mathrm{PFP}^{12}$ ) and $\Delta$ is the environmental contribution accounting for intermolecular (electrostatics, $\Delta_{E}$, and induction, $\Delta_{I}$ ) and substrate interactions. The environmental terms $\Delta$ have been evaluated with the CR model ${ }^{51,52}$ as implemented in the 
MESCAL code. ${ }^{53} \mathrm{CR}$ calculations were parametrized with DFT (PBE0/6-311++G** level) polarizability and ESP charges, ${ }^{38}$ and semiempirical (ZINDO) atom-atom polarizability tensor. ${ }^{51}$ All results have been extrapolated to the infinite film limit.

PEN:PFP films on $\mathrm{SiO}_{\mathrm{x}}$ and $\mathrm{HOPG}$ were modeled using the substrate-specific atomistic crystal structures determined in the present study. Films on $\mathrm{SiO}_{\mathrm{x}}$ have been modeled as free-standing bilayers, while the interaction with the conducting HOPG substrate has been described with an image charge model. The $\left(\begin{array}{lll}1 & 0 & 0\end{array}\right)$ crystal facet has been employed in calculations of PEN:PFP films with standing molecular orientations. Calculations for PEN:PFP heterostructures on HOPG considered films with (-1 -2 1) orientation and different thickness; the crystal structures of pure PEN and PFP phases were taken from the literature. ${ }^{25,26,42,54}$ See the Supporting Information (SI) for further details.

\section{Results and Discussion}

\section{Experimental Structure Determination}

As the IP of organic thin films depends on the molecular orientation, ${ }^{18,19}$ we have first performed specular X-ray diffraction (XRD) on PEN:PFP co-deposited films of 1:1 stoichiometry on HOPG (Fig. 2a). The only diffraction features of the organic adsorbate are two reflections (labeled L and L') close to the (0 02) peak of the HOPG substrate, which correspond to lattice spacings of $3.14 \AA(\mathrm{L})$ and $3.26 \AA\left(\mathrm{L}^{\prime}\right)$, respectively. Notably, these values are close to that of $3.07 \AA$ which we observed for the stacking distance of pristine PFP on HOPG, as grown in a flat-lying $\pi$-stacked polymorph ${ }^{25}$ In analogy, we therefore expect these reflections to be due to lying molecules as well in the mixed film. The occurrence of two reflections instead of one (both corresponding to a similar lattice spacing) can either point to the growth of two orientations of one single phase, or to the presence of two slightly different mixed polymorphs. Tentatively assuming the latter, we changed our preparation protocol from co-deposition to the sequential deposition of alternating 

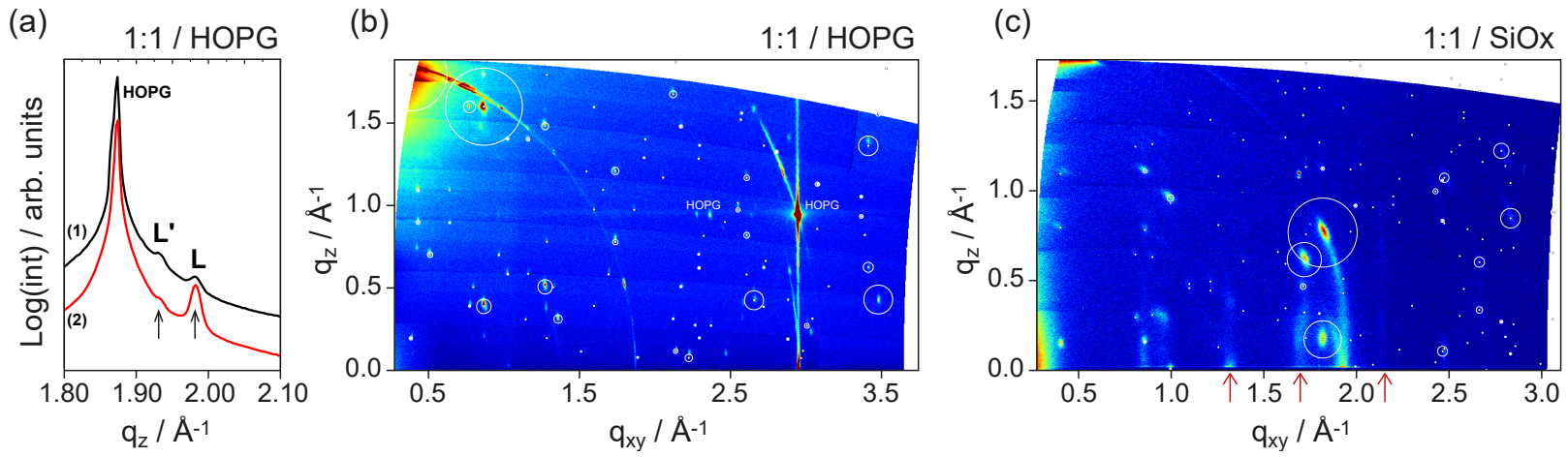

Figure 2: (a) XRD on nominally $300 \AA$ thick films of 1:1 co-deposited PEN:PFP (curve 1) and of 75 alternating layers of PFP and PEN with a nominal thickness of $4 \AA$ each (curve 2). Two diffraction features $\mathrm{L}$ and $\mathrm{L}^{\prime}$ are observed close to the strong (002) reflection of HOPG; curves are vertically shifted for clarity. (b) GIXRD reciprocal space map of the film obtained by layered deposition (curve 2 in (a)). Reflections labeled with $H O P G$, the vertical feature at ca. $q_{x y}=3 \AA^{-1}$, and diffraction intensity on rings of constant $q=\sqrt{q_{z}^{2}+q_{x y}^{2}}$ stem from the HOPG substrate. (c) Reciprocal space map of a nominally $300 \AA$ thick film of co-deposited PEN:PFP on $\mathrm{SiO}_{\mathrm{x}}$; red arrows indicate positions of weak vertical diffraction features along $q_{z}$ which cannot be explained by the pure materials and are likely due to an additional upright-standing mixed phase of PEN:PFP. ${ }^{28,32}$ In both figures, areas of the white circles correspond to calculated intensities for our structure solutions.

monolayers (starting with nominally $4 \AA$ of PFP) to finally reach the same overall nominal thickness of $300 \AA$; the corresponding XRD data is shown in Fig. 2(a) as curve 2. Clearly, diffraction feature $L^{\prime}$ is now almost entirely attenuated, while feature L is largely enhanced in intensity. As relative diffraction intensities are, in general, defined by the crystal structure itself, the two diffraction features $\mathrm{L}$ and L' represent two different polymorphs, where that corresponding to $\mathrm{L}$ is severely promoted by sequential deposition (see SI for a comparison of GIXRD data). As L corresponds to the lower lattice spacing, we expect this structure to represents a more closely packed, mixed crystalline structure of PEN and PFP.

Motivated by the significantly enhanced scattering intensity of this film, we performed GIXRD on the sample. On amorphous substrates like $\mathrm{SiO}_{\mathrm{x}}$ which promote fibre-textured growth of the organic adsorbate (i.e., growth in the form of a two-dimensional powder), a single reciprocal space map from GIXRD can provide, in principle, sufficient information 
of the crystal structure for deriving a structure solution. In contrast to single crystalline substrates, this is equally true for HOPG surfaces promoting quasi fibre-textured growth of the organic adsorbate, because the statistically oriented single-crystalline graphite grains on the substrate surface (with lateral sizes in the $\mu \mathrm{m}$ range) are far smaller than the footprint of the X-ray beam $\left(\geq 1 \mathrm{~mm}^{2}\right)$. The reciprocal space map in Fig. $2 \mathrm{~b}$ for the film established by layered deposition (curve 2 in Fig. 2a) can therefore be readily used for determining the crystal structure by GIXRD. Inspired by the general procedures for indexing experimental GIXRD patterns of organic thin films recently reported by some of us, 55,56 we determined the crystallographic unit cell from the experimental peak positions. We find triclinic unit-cell parameters of $a=15.72 \AA, b=7.41 \AA, c=7.25 \AA, \alpha=102.2^{\circ}$, $\beta=67.45^{\circ}$, and $\gamma=98.0^{\circ}$ (uncertainty of $\pm 0.05 \AA$ on lattice parameters and of $\pm 0.2^{\circ}$ on angles) grown in (-1 -21) orientation on the HOPG substrate.

The plausibility of this unit cell is best seen from the reciprocal space map of a 1:1 co-deposited film on $\mathrm{SiO}_{\mathrm{x}}$, as shown in Fig. 2c. For this system, a reflection in XRD corresponding to a lattice spacing of $6.64 \AA$ has been the first evidence for 1:1 mixed crystal growth in previous studies. ${ }^{18,28}$ By slightly refining above unit-cell parameters determined for the film on the HOPG substrate to $a=15.68 \AA, b=7.38 \AA, c=7.25 \AA$, $\alpha=102.8^{\circ}, \beta=68.6^{\circ}$, and $\gamma=97.6^{\circ}$, we can index all peaks in the reciprocal space map, now for $(001)$ orientation on the $\mathrm{SiO}_{\mathrm{x}}$ substrate. Merely, the significantly different angle $\beta$ indicates that a (slightly) different polymorph of the mixed film grows on $\mathrm{SiO}_{\mathrm{x}}$. As an aside we note that, in addition to the 1:1 mixed crystalline phase, these films further comprise a less-well ordered phase of standing molecules (likely corresponding to weak diffraction features marked with red arrows in Fig. 2c), which, likewise, have been regarded to be intermixed on the molecular scale. ${ }^{18}$ 


\section{Full Structure Solution by Modeling}

Both pure materials show pronounced polymorphism and a number of different crystal structures have been reported for PEN to date, all of which show a layered structure with herringbone molecular packing. ${ }^{40,41,57-60}$ The same is true for PFP, both in its single-crystal phase ${ }^{42}$ and in thin films grown on $\mathrm{SiO}_{\mathrm{x}},{ }^{25}$ while, instead, we found $\pi$-stacked growth of PFP both on HOPG and graphene in a previous study. ${ }^{25}$ All crystal forms of pure PEN and PFP exhibit structures with two molecules in the unit cell, sitting on inversion sites; as the PFP molecule has slightly larger van der Waals volume than PEN, its unit cells are accordingly larger. In analogy to the herringbone structures prevailing for both materials it has been suggested that the PEN:PFP mixed crystal structure would crystallize, likewise, in a herringbone motif forming a unit cell comprising two molecules. ${ }^{29,61}$ Following the approach we successfully applied in previous studies, ${ }^{7,25,62-64}$ we provide here a full structure solution of the 1:1 mixed phase by modeling the molecular arrangement in the experimentally determined unit cell. This provides numerous candidate structures that are then confirmed (or excluded) by comparing the computed diffraction intensities with the experimental ones.

The experimental unit cells of the mixed phases on $\mathrm{SiO}_{\mathrm{x}}$ and HOPG substrates are triclinic, and, thus, compatible only with space groups $P 1$ (no symmetry) or $P \overline{1}$ (inversion symmetry only). Because the PEN and PFP molecules are both centrosymmetric, and as molecules usually maintain their inversion symmetry when forming a crystal, ${ }^{65}$ we expect both PEN:PFP phases to crystallize in space group $P \overline{1}$, with molecules sitting on inversion sites. The unit-cell volumes deduced from GIXRD are intermediate between the respective volumes for $\mathrm{PEN}^{40,41,57-60}$ and $\mathrm{PFP},{ }^{25,42}$ which is in line with what one would expect for a crystal containing two molecules in the unit cell, one of each species.

Having chosen and validated the potential model on the pure compounds (see Experimental Section for details), we have generated over 30000 initial structures for the PEN:PFP mixed crystal. The unit-cell parameters were fixed to the experimental values, 
the PEN molecule at the origin, and the PFP molecule at any of the remaining inversionequivalent inversion sites. These restrictions significantly constrain the possible crystal structures, making a systematic search feasible. The initial quasi-random configurations were optimized by minimizing the lattice energy with respect to the molecular orientations for fixed lattice parameters and molecular center-of-mass positions, imposed by the symmetry, and finally ranked by energy. The deepest PEN:PFP constrained minimumenergy structure for the unit cell on HOPG is depicted in Fig. 3a-c. Its calculated X-ray diffractogram reproduces well the observed intensity pattern of the reciprocal space map in Fig. 2b. Note that all other, more shallow, minima yield unreasonable diffraction intensities. In the minimum-energy structure, PEN and PFP molecules are packed in alternating stacks along the $b$-crystal axis, with an intermolecular spacing $b / 2$ of $\approx 3.7 \AA$; the angle between the PEN and PFP molecular planes is just $4.35^{\circ}$. Notably, for the slightly different unit cell deduced from the GIXRD data on $\mathrm{SiO}_{\mathrm{x}}$ (Fig. 2c) we find an essentially identical crystal structure following the same approach; both crystallographic information files (CIFs) are provided as SI. We note that such a cofacial mixed-stack packing of these molecular species with opposite quadrupole moments is highly plausible due to their stabilizing electrostatic interactions.

Our experimental data (XRD and GIXRD) indicate growth of the adsorbate in (-1 -21) texture on HOPG, i.e., this specific crystallographic plane of the PEN:PFP crystal structure (Fig. 3d) is parallel to the substrate surface (i.e., the HOPG (0 0 1) plane). The long axes of PEN and PFP are inclined by less than $5^{\circ}$ with respect to the substrate in the bulk film; the molecules therefore lie essentially parallel to the substrate and pile up in alternating stacks perpendicular to it. We note that comparing the C-C spacing within the HOPG (001) plane and the lateral molecular spacing of PEN and PFP within the (-1 21) plane reveals remarkable commensurability of these structures (see SI). During initial film growth, we therefore expect the first adsorbed molecules to adopt an arrangement already largely similar to that in the mixed crystal structure, likely in a flat-lying orienta- 
(a)

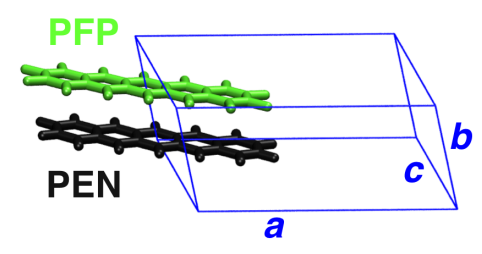

(c)

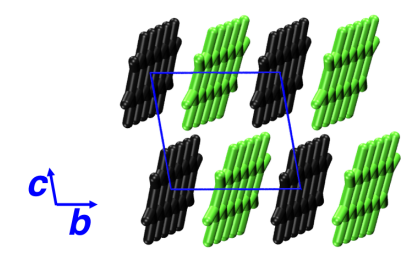

(b)

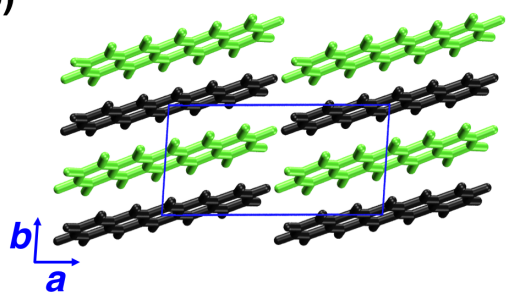

(d)

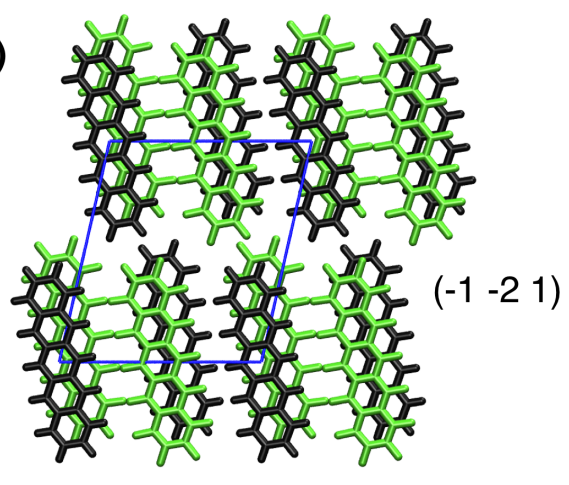

Figure 3: (a-c) Illustrations of the 1:1 crystal structure of PEN:PFP (black-green color) grown on HOPG. An almost identical unit cell was determined for the $\mathrm{SiO}_{\mathrm{x}}$ substrate. (d) Top-view on the (-1 -21) plane, which was found as texture plane on HOPG (i.e., lies parallel to the HOPG (0 01$)$ plane).

tion, as we have already found for the pristine PFP film on graphene and HOPG before. ${ }^{25}$ Such an initial molecular arrangement in the monolayer is already close to that of the bulk minimum-energy structure is expected to subsequently promote its growth in the three-dimensional film. ${ }^{10}$

\section{Band Structure of the PEN:PFP Co-crystal}

Thin films of pristine PEN and PFP as well as PEN single crystals have been experimentally shown to exhibit pronounced band dispersion. ${ }^{25,27,66,67}$ As the structure solution for the mixed crystal reveals cofacial PEN and PFP molecules, its electronic structure might, in principle, be strongly affected by the formation of band states as well, possibly including some hybridization between PEN and PFP orbitals mediated by a pronounced wavefunction overlap, which would explain the ultra-low IP of the mixed film (cf. Fig. 1c)..$^{5,68,69}$ To 
explore this possibility, we computed the band structure of the co-crystal with periodic density functional theory (DFT) calculations. The band structure shown in Fig. 4 (exemplary determined for the structure grown on HOPG) reveals that the valence states are characterized by two nearly flat and non-overlapping bands. The illustration of the density of states (DOS) in the right panel shows the contribution of PEN (black) and PFP (green) atomic orbitals to the total DOS (red line) and reveals that the highest-energy valence band is mostly composed of PEN HOMOs, while PFP HOMOs mostly contribute to the second band. A very similar situation is observed for conduction states, with well distinguishable bands of PEN and PFP LUMOs. The maximal dispersion occurs, as expected, along the $\pi$-stacking direction. The small bandwidth $(\sim 0.1 \mathrm{eV})$ can be explained by a superexchange mechanism in which hoppings are mediated by the molecule in between,$^{70}$ consistently with the sizeable charge transfer integrals between the frontier orbitals of neighboring PEN and PFP molecules, reported in Table 1. The same appreciable couplings are also responsible for the appearance of a charge-transfer excitation in the optical absorption spectrum of PEN:PFP blends. ${ }^{10,30}$

Overall, the picture emerging from periodic DFT calculations is that of very narrow bands presenting only weak intermolecular hybridization. This result rules out that the new features in the photoemission spectra of the mixed film, and the IP lower than that of the pristine materials in particular, arise from orbital hybridization. ${ }^{5,68,69}$ We can therefore safely consider charge carriers to be localized on molecular units at room temperature, as a result of the energetic disorder due to thermal lattice motion.

Table 1: Charge transfer integrals (absolute values in $\mathrm{meV}$ ) between the frontier energy levels of neighboring PEN and PFP molecules.

\begin{tabular}{ccc} 
& PFP-HOMO & PFP-LUMO \\
\hline \hline PEN-HOMO & 80.9 & 29.8 \\
\hline PEN-LUMO & 22.9 & 8.3 \\
\hline \hline
\end{tabular}




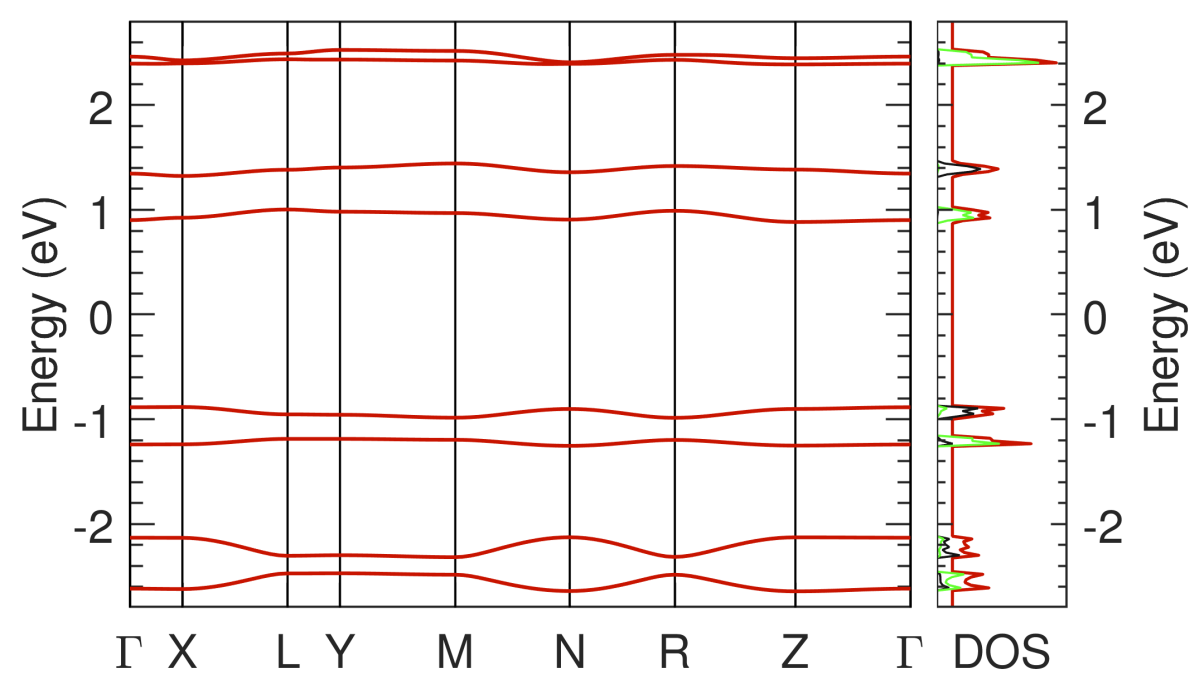

Figure 4: Calculated band structure of the PEN:PFP mixed crystal (structure determined for the HOPG substrate). Frontier orbitals (HOMO and LUMO) of PEN and PFP molecules result in flat bands, pointing to localized charge carriers at room temperature. The right panel shows the total density of states (DOS, red line) and the partial DOS obtained by summing up the contributions from atomic orbitals of PEN (black line) and PFP (green line) molecules. The high-symmetry points of the Brillouin zone are: $\Gamma=\left(\begin{array}{lll}0 & 0 & 0\end{array}\right)$, $X=\left(\begin{array}{lll}0.5 & 0 & 0\end{array}\right), L=\left(\begin{array}{lll}0.5 & 0.5 & 0\end{array}\right), Y=\left(\begin{array}{lll}0 & 0.5 & 0\end{array}\right), M=\left(\begin{array}{lll}0 & 0.5 & 0.5\end{array}\right), N=\left(\begin{array}{lll}0.5 & 0 & 0.5\end{array}\right)$, $R=\left(\begin{array}{lll}0.5 & 0.5 & 0.5\end{array}\right), Z=\left(\begin{array}{lll}0 & 0 & 0.5\end{array}\right)$.

\section{Modeling UPS Spectra of PEN:PFP Films of Standing Molecules}

The UPS spectra of mixed PEN:PFP films on $\mathrm{SiO}_{\mathrm{x}}$ are depicted in Fig. $1 \mathrm{~b}$ along with the valence band spectra of pure PEN and PFP films (data taken from Ref. 18). Two low-energy peaks at binding energies between those of pristine materials are found, which have been interpreted as resulting from the superposition of the opposite quadrupole moments of PEN and PFP (see Fig. 1a). ${ }^{18}$ A more detailed modeling of these UPS spectra was later attempted by Topham and Soos. ${ }^{35}$ Because of the unknown structure of the mixed crystal, they had to assume an hypothetical packing obtained by substituting molecules in the herringbone structures of the neat components. Their results highlighted the strong 
dependence of the IP (5.44-5.99 eV range for PEN, 6.26-6.67 eV range for PFP) on fine details of the herringbone packing and, therefore, could not provide reliable quantitative agreement with the experiment.

Here, we can now take advantage of our structure solution for the mixed polymorph on $\mathrm{SiO}_{\mathrm{x}}$ (see above) to attempt a quantitative modeling of these UPS data based on atomistic charge response (CR) model calculations. Our modeling considers films of standing molecules built from our crystal cell $\left[\begin{array}{lll}1 & 0 & 0\end{array}\right)$ face $]$, assuming that the mixed-stack structure is representative for the whole mixed film; technical details are provided in the Experimental Section and in the SI. The results of our calculations for both pure and mixed films are shown as vertical bars in Fig. 1b. They agree within $0.2 \mathrm{eV}$ with the experimental peaks in the UPS spectra. For pure films of standing PEN or PFP molecules, our results are fully consistent with previous calculations. ${ }^{16,20,35}$ In pure PEN films the electron binding energy is reduced when going from the gas phase to the solid state (see Table 2). Conversely, in pure PFP films the values for the molecule in the gas phase and in the solid state are rather similar. The doublet observed between $6.2 \mathrm{eV}$ and $6.8 \mathrm{eV}$ for the mixed film (red curve in Fig. 1b) is also well reproduced by our calculations. In particular, the lowest binding energy peak can be assigned to the ionization of PEN (black bar), while the deeper level corresponds to PFP (green bar).

Deeper insight in the charge-carrier energetics can be gained from partitioning contributions of the surrounding on energy-level positions into induction and electrostatics, as reported in Table 2 (see Experimental Section). ${ }^{14}$ Induction interactions are always stabilizing the hole, which reduces the IP by a similar amount in virtue of the generally similar dielectric constants of organic solids $\left(\varepsilon_{r} \sim 3-4\right)$. Instead, the relationship between molecular organization and energy levels is mostly governed by the electrostatic contribution. Electrostatic shifts in the mixed structure are smaller than in pristine systems and have opposite sign for the ionization of PEN and PFP. Since both molecules feel the same longrange electrostatic environment, determined by the negligible quadrupole moment of the 
1:1 crystal unit cell (the quadrupoles of cofacial PEN and PFP cancel out almost exactly, see Fig. 1a), these differences can be attributed to short range interactions. In fact, in the mixed-stack packing, each PEN molecule is sandwiched between two PFP molecules and a hole localized on PEN is destabilized by the quadrupole moments of the neighboring PFP molecules presenting an out-of-plane component of positive sign. ${ }^{14}$ The opposite situation is experienced by a hole on PFP, that is instead stabilized by the interaction with the neighboring PEN molecules, hence, shifting the corresponding peak to lower binding energy. Overall, blending dissimilar organic compounds on the molecular scale permits tuning the energy levels due to an averaging of the molecular multipole moments. 3,15,18 However, as we will see in the following, this simple interpretation is not equally true for every molecular orientation.

Table 2: Partitioning of CR calculation results in Fig. 1a into electrostatic $\left(\Delta_{E}\right)$ and induction $\left(\Delta_{I}\right)$ components (see Experimental Section). Energies are in $\mathrm{eV}$. The induction components always stabilize the hole by approximately $0.7-0.8 \mathrm{eV}$, while the electrostatic term is highly sensitive to the molecular packing.

\begin{tabular}{lccc} 
system & $\Delta_{E}$ & $\Delta_{I}$ & $\mathrm{IP}$ \\
\hline \hline PEN & -0.42 & -0.82 & 5.35 \\
PFP & 0.64 & -0.70 & 7.45 \\
PEN:PFP & 0.45 & -0.69 & 6.35 \\
PEN:PFP & -0.27 & -0.67 & 6.56 \\
\hline \hline
\end{tabular}

\section{UPS Spectra of PEN:PFP Films of Lying Molecules on HOPG}

We now turn to the electronic structure of PEN:PFP mixed films on the HOPG substrate in lying orientation. Fig. 5 compares the UPS spectra of pure PEN and PFP monolayers, bilayers (PEN/PFP and PFP/PEN) obtained via sequential deposition, and equimolar mixed films of increasing nominal thickness grown by 1:1 co-deposition. The IP values of pure monolayer PEN (5.5eV) and PFP (5.9 eV) films are well in line with previous reports for these materials, both on HOPG $23,71,72$ and on metal substrates. ${ }^{73}$ The scenario emerging for mixed PEN:PFP films on HOPG is, however, significantly more complex than on $\mathrm{SiO}_{\mathrm{x}}$ 


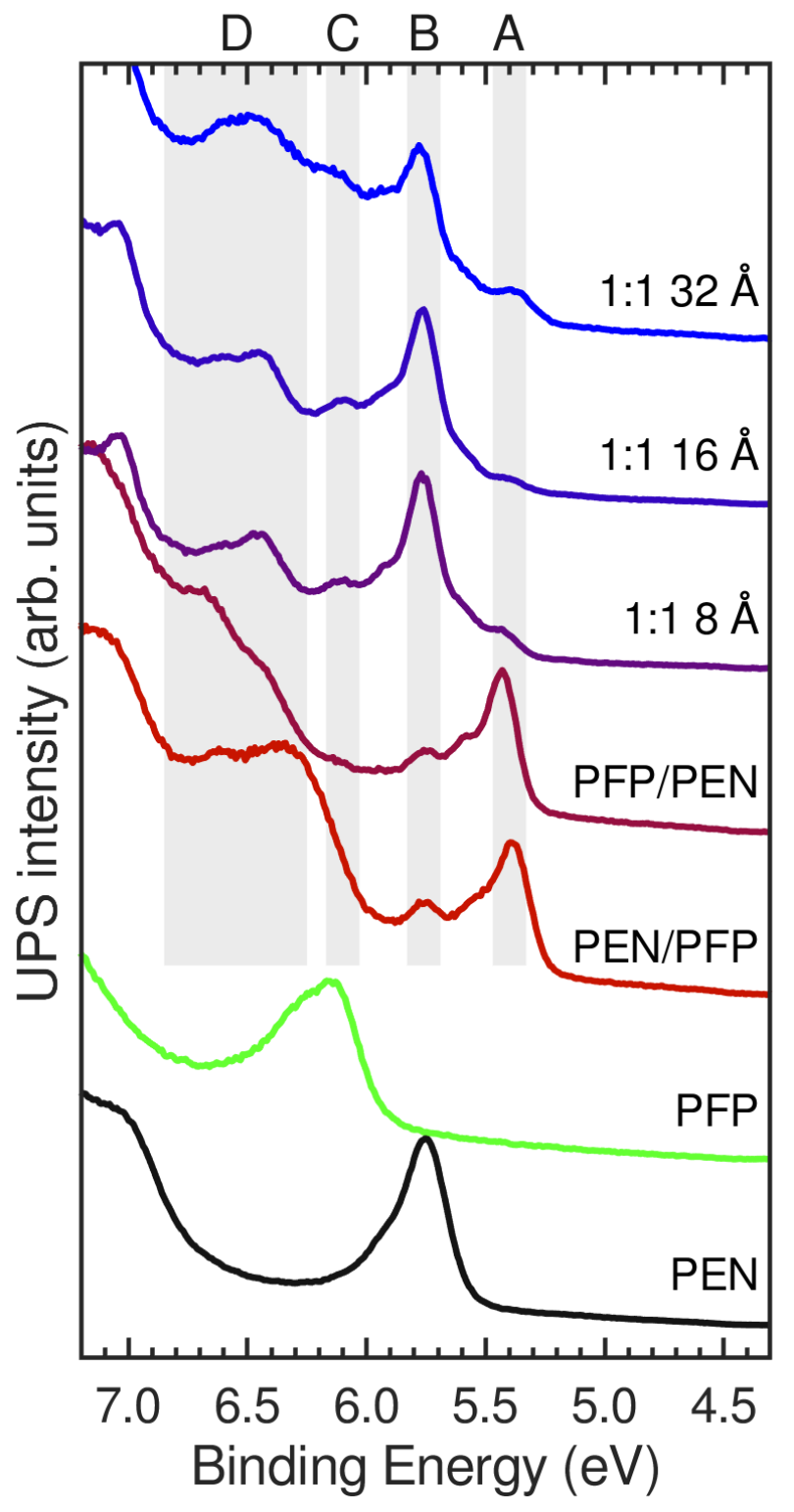

Figure 5: Experimental UPS spectra of films of lying orientation on HOPG. Spectra include bilayers obtained by sequential deposition of PEN on PFP (PEN/PFP) and vice versa (PFP/PEN), as well as 1:1 films obtained by co-deposition of different thickness; the $8 \AA$ spectrum is also shown in Fig. 1c. The spectra of PEN and PFP monolayers (black and green lines, nominal thickness of $4 \AA$ ) are shown as reference. Labels A-D highlight specific spectral features discussed in the main text. 
(cf. Fig. 5 and Fig. 1b), as several UPS features occur in the valence-electron region at BEs below $7 \mathrm{eV}$. The lowest BE peak of the mixed films (labeled as A in Fig. 5) translates into an IP of $5.3 \mathrm{eV}$. This value does not lie in between those of pristine PEN and PFP, as it has been the case for the standing films on $\mathrm{SiO}_{\mathrm{x}}$ before, but is now lower than both. Further, it is found to be essentially independent of the film thickness up to $32 \AA$, which corresponds to nominally 8 molecular layers in lying orientation.

Interestingly, regardless of the deposition sequence, the spectra of both bilayers are highly similar to each other. As UPS is very surface sensitive, this points to an identical film structure forming in both cases, which is perfectly in line with our GIXRD result of mixed crystal growth upon sequential deposition ( $c f$. Fig. 2b). The most intense peak is centered at $5.4 \mathrm{eV}$ (A in Fig. 5), the second most intense at $5.8 \mathrm{eV}$ (B), with a third, weaker feature in between. Also in the energy range around $6.5 \mathrm{eV}$ labeled as $\mathrm{D}$ in Fig. 5 the UPS data of both bilayers exhibit similar features. Likewise, thicker films obtained by co-deposition show photoemission features at these positions (see spectra of 8-32 $\AA$ nominal thickness in Fig. 5), albeit the relative intensities are remarkably different from the bilayers. The spectra of the co-deposited films are dominated by feature B instead of A, which is still there but significantly weaker than in the bilayers. We also notice the presence of another peak at $6.1 \mathrm{eV}(\mathrm{C})$ which was not observed in the spectra of the bilayers.

Importantly, the signal of the HOPG substrate is present in all spectra, even for films of nominal thickness beyond $3 \mathrm{~nm}$ (see SI for full spectra). This indicates an inhomogeneous growth of the film, where regions of the substrate are either bare or covered by one molecular layer (ML) only. We note that so-called island or Stranski-Krastanov (layer plus island) growth modes are very common for vacuum-deposited thin films of such materials ${ }^{74-76}$ and were, in particular, shown for PEN on HOPG. ${ }^{77}$

The more complex scenario emerging from the UPS spectra of the mixed films on HOPG as compared to the $\mathrm{SiO}_{\mathrm{x}}$ case immediately raises a number of questions: (i) what 
is the origin of the ultra-low IP of $5.3 \mathrm{eV}$ of the mixed films? To the best of our knowledge, this value is by far the lowest IP ever reported for PEN or PFP in lying orientation. (ii) What is the reason for the marked difference between the UPS spectra of nominal bilayers and the (thicker) co-deposited films? (iii) Why are the UPS spectra of lying, mixed films so rich in features as compared to the $\mathrm{SiO}_{\mathrm{x}}$ case ${ }^{15,18}$ which only shows two low $\mathrm{BE}$ peaks, as it would be expected for a two-component system?

\section{Modeling UPS Spectra of PEN:PFP Films of Lying Molecules}

To answer these questions, we have performed a series of theoretical CR calculations of the photoemission energy levels. Following Soos and coworkers, ${ }^{35,78}$ the effect of the conducting substrate has been taken into account with an image-charge model. The results of CR calculations for pure PEN and pure PFP monolayers of lying molecules on HOPG are in very good agreement with the experimental UPS (see Fig. 1c), which validates the proposed approach that we now apply to PEN:PFP mixed films. We have therefore considered PEN:PFP films of lying molecules with a thickness of one to four MLs. We have explicitly considered the ionization of molecules in each layer, as we expect the experimental UPS signal to not only originate from molecules at the surface, but also, to some extent, from buried molecules. This is justified as the thickness of a layer of lying molecules (ca. $4 \AA$ ) is, in fact, in the range of the electron mean free path in the medium, which can be deduced from the so-called universal curve by Seah and Dench ${ }^{79}$ to be ca. $7 \AA$ for our experimental conditions.

The calculated UPS spectra of PEN:PFP films on HOPG are shown in Fig. 6 as a function of the film thickness, the IPs of molecules located in the different layers are plotted as vertical bars. Assuming a simple exponential decay of the photoemission intensity with depth (decay length of $7 \AA$, i.e the electron mean free path), the length of the bars then provides a rough estimate for the expected UPS intensity. The calculated spectrum for the 1 ML film shows two peaks that correspond to the ionization of PEN (black bar 


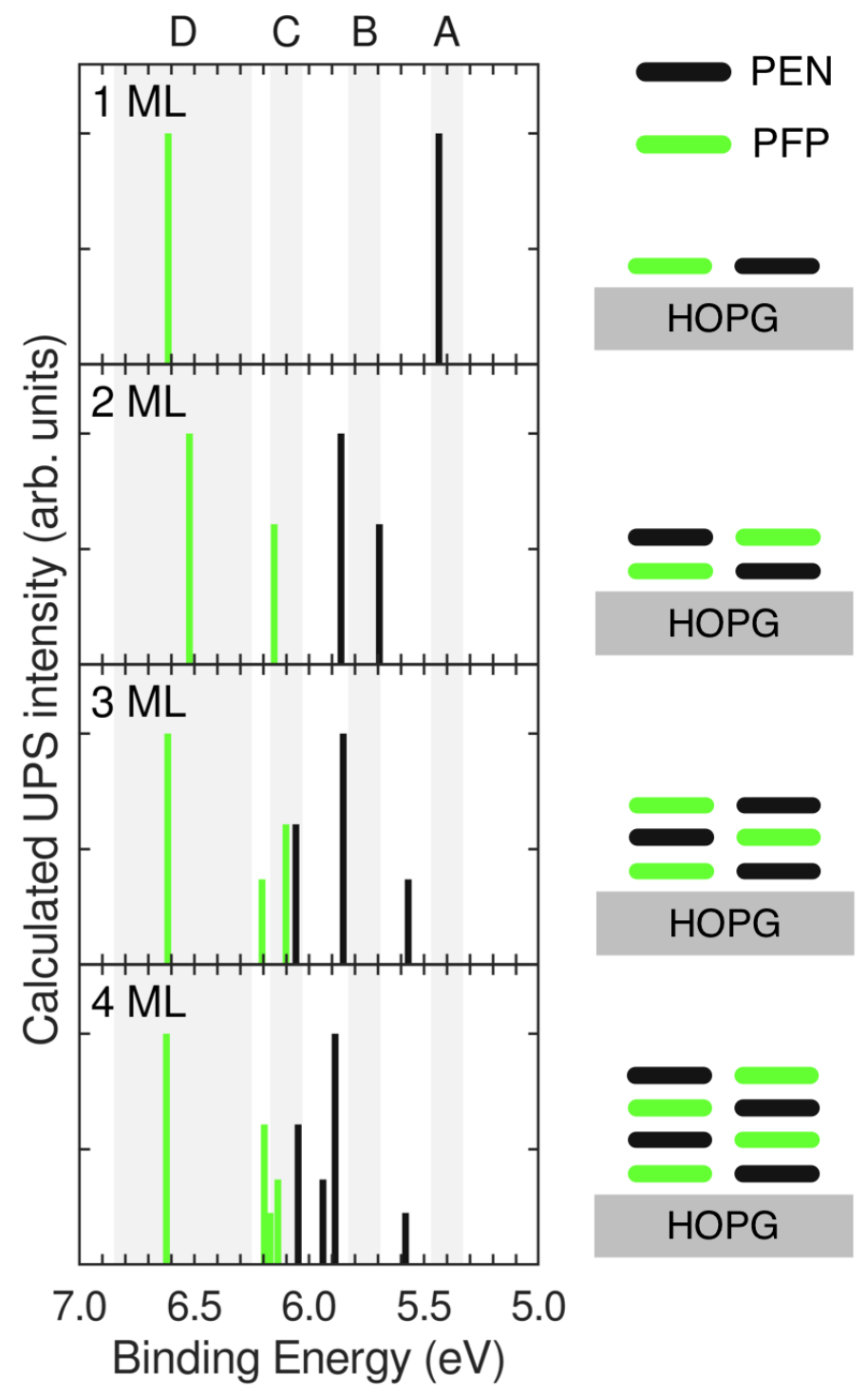

Figure 6: Calculated UPS spectra of PEN:PFP films of 1-4 molecular layers (ML); black an green bars correspond to the IPs of PEN and PFP molecules, respectively. The calculated intensity (bar height) depends on the depth $z$ of the ionized layer from the surface as $\exp (-z / \lambda)(\lambda=7 \AA)$, so that most intense peaks always correspond to the ionization of molecules at the film surface. Note the remarkable difference between the spectra of PEN:PFP monolayer (1 ML) and multilayers (2-4 ML). 
at $5.44 \mathrm{eV}$ ) and PFP (green bar at $6.61 \mathrm{eV}$ ). We immediately notice that the PEN feature is in very good agreement with the experimental UPS peak A (Fig. 5), from which the surprisingly low IP of the mixed films has been deduced. For mixed multilayers, we find that the calculated energy of the most intense peak of PEN and PFP, which corresponds to molecules at the surface, is essentially independent of the number of layers (2-4 ML). The IP of PEN at the surface is, e.g. for $3 \mathrm{ML}$, calculated to $5.85 \mathrm{eV}$, a value that is consistent with the position of peak B in the experimental UPS data, which we recall to be the dominating feature in the co-deposited films of $\geq 8 \AA$ nominal thickness. The maybe most remarkable finding is the ca. $0.4 \mathrm{eV}$ difference in IP between PEN in the monolayer (1 ML) and at the surface of multilayers (2-4 ML).

To understand the origin of this equally large and unexpected difference, we have partitioned the calculated IP into its different contributions, i.e., electrostatics, induction, and substrate interactions - a detailed analysis of the thickness and layer dependence of the different terms is provided as SI. We find that most of the thickness dependence of the IP in the present films of lying molecules arises from the electrostatic term $\left(\Delta_{E}\right)$, which itself strongly depends on the molecular organization. Induced polarization in the organic film and image charges play a minor role, since these two effects roughly compensate each other (see SI). Specifically, in the $1 \mathrm{ML}$ case the molecules are arranged laterally side-byside, as illustrated in Fig. 3d. Therefore, any given PEN molecule is surrounded by the fluorine atoms of neighboring PFP molecules, which bear a negative partial charge. The relevant components of the molecular quadrupoles (see Fig. 1a) are therefore the in-plane components, which laterally stabilize a hole in $\operatorname{PEN}\left(\Delta_{E}=-0.30 \mathrm{eV}\right)$. On the other hand, in multilayer films, molecules stack along the plane normal with an alternating motif, as shown in Fig. 3 and Fig. 6 . The vertical interaction between $\pi$-stacked molecules of different nature is governed by the out-of-plane components of the molecular quadrupoles, which have opposite signs than the in-plane components (see Fig. 1a). This is ultimately responsible for the IP difference between mono- and multilayers. 
The features in the $6.3-6.8 \mathrm{eV}$ window of the experimental UPS data (labeled as D in Fig. 5) are broadly consistent with the ionization of PFP surface molecules in $1 \mathrm{ML}$ as well as in multilayer films. In this energy region, however, an unambiguous assignment of experimental features is no longer possible, because of the several peaks expected in a narrow energy window ( $c f$. Fig. 6) and because PEN HOMO-1 related photoemission is expected at $1.3 \mathrm{eV}$ BE above the PEN HOMO. ${ }^{49,80}$ Finally, the calculated spectra of the 2-4 ML films further show a series of weaker peaks due to sub-surface molecules that occur at significantly different energies than the main feature. A series of peaks are predicted around $6.1 \mathrm{eV}$, that is, in the spectral region of peak $\mathrm{C}$ in the experimental UPS data in Fig. 5.

\section{Conclusion}

Our calculation methodology accurately reproduces the main features found in the UPS spectra of PEN:PFP, both for films on $\mathrm{SiO}_{\mathrm{x}}$ and HOPG. In contrast to previous studies, our present calculations have been performed under the precise knowledge of the underlying structure, where the 1:1 co-crystals are characterized by one-dimensional stacks of cofacially alternating PEN and PFP molecules (mixed-stack motif). While the mixed crystal structures grown on $\mathrm{SiO}_{\mathrm{x}}$ and $\mathrm{HOPG}$ are found to be almost identical, their different molecular orientation is dictated by the nature of the substrate, which entails crucial consequences for the electronic structure of the adsorbate. For PEN:PFP films on $\mathrm{SiO}_{\mathrm{x}}$ grown in standing molecular orientation, our calculations yield an IP value between the values of the two neat components (Fig. 1b). This is a result of the averaging of the quadrupole moments of standing molecules and is in line with previous studies that required assuming hypothetical structures. ${ }^{3,15,18,35}$

Understanding the significantly more complex scenario observed for mixed films of lying molecules on HOPG (Fig. 5), however, required the precise knowledge of the pack- 
ing. Therefrom we deduced a conclusive scenario for the interplay between structure, morphology and electronic properties of the mixed films, which allowed us to assign experimental UPS features to PEN and PFP molecules in films of different thickness. Therefrom, the following growth scenario emerges naturally: Stranski-Krastanov growth of the films occurs, which is characterized by the coexistence of substrate regions covered with a mixed monolayer and regions with mixed multilayer islands. ${ }^{74}$ Therefore, the experimental UPS data represent the superposition of the signals of mono- and multilayers, in proportions that depend on the nominal film thickness. The UPS spectra of ultra-thin films (i.e., the nominal bilayers PEN/PFP and PFP/PEN) are dominated by the photoemission of the monolayer, which leads to the surprisingly low IP value of $5.3 \mathrm{eV}$ (peak A in Fig. 5). According to our calculations this is due to the in-plane components of the molecular quadrupoles in the lateral packing specific to the mixed layer of lying molecules. In addition, the distinctive signal of the multilayer (peak B in Fig. 5) is already detected in this case due to the specific growth mode, however, only with low intensity. This peak corresponds to a significantly higher IP of $5.7 \mathrm{eV}$ and can be regarded as fingerprint of the multilayer, as it clearly dominates the valence band UPS spectra of thicker films established by co-deposition. Notably, in these cases peak A still remains detectable for all investigated thicknesses, as a result of the Stranski-Krastanov growth mode.

In summary, our study discloses the role that both intermolecular and substrate interactions can play in determining the molecular organization and the ensuing electronic structure of thin films composed of organic semiconductor blends. The spatial proximity of different molecular quadrupoles in such films-brought about by compounds comprising strong intramolecular polar bonds-can severely modify the energy positions of the frontier molecular energy levels as a function of the molecular position in the film. For the case of the prototypical organic semiconductors PEN and PFP, our study reveals that this effect is particularly important for lying molecules in molecularly mixed films. This orientation is not only commonly found on graphene and graphite, but also on metal- 
lic substrates generally used as electrodes in practical applications. As thusly modified energy levels define the relevant energies for charge injection into organic electronic devices, e.g., the hole injection barrier as defined by the IP, blends of dissimilar organic semiconductors may provide a novel means of control for such barriers at interfaces between functional layers and electrodes. The insights gained through our comprehensive structural analysis and electronic characterization of this prototypical system represent a step towards establishing robust structure-property relationships for the rational design of novel organic functional materials.

\section{Associated Content}

Supporting Information. GIXRD data of a 1:1 co-deposited PEN:PFP, full range UPS data, illustration of the commensurism between the 2D lattice of the HOPG (001) plane and the (-1 -21) plane of PEN:PFP, details on the CR model calculations, Crystallographic Information Files (CIFs) of the PEN:PFP mixed crystal polymorphs on $\mathrm{HOPG}$ and $\mathrm{SiO}_{\mathrm{x}}$ substrates. The Supporting Information is available free of charge on the ACS Publications website.

\section{Acknowledgements}

Financial support from the 111 Project of the Chinese State Administration of Foreign Experts Affairs, the Collaborative Innovation Center of Suzhou Nano Science \& Technology (NANO-CIC) and the Soochow University-Western University Joint Center for Synchrotron Radiation Research is gratefully acknowledged. We thank Wolfgang Caliebe (HASYLAB, DESY) for experimental support and Roland Resel (TU-Graz) for fruitful discussions. I.S. acknowledges support of the Natural Sciences and Engineering Research Council of Canada (NSERC) [funding reference number RGPIN-2018-05092], the Fonds 
de recherche du Québec - Nature et technologies (FRQNT) [funding reference 2020-NC271447], and by Concordia University. 


\section{References}

(1) Ostroverkhova, O. Organic Optoelectronic Materials: Mechanisms and Applications. Chem. Rev. 2016, 116, 13279-13412.

(2) Clarke, T. M.; Durrant, J. R. Charge Photogeneration in Organic Solar Cells. Chem. Rev. 2010, 110, 6736-6767.

(3) Heimel, G.; Salzmann, I.; Duhm, S.; Koch, N. Design of Organic Semiconductors from Molecular Electrostatics. Chem. Mater. 2011, 23, 359-377.

(4) Yang, J.-P.; Bussolotti, F.; Kera, S.; Ueno, N. Origin and role of gap states in organic semiconductor studied by UPS: as the nature of organic molecular crystals. J. Phys. D: Appl. Phys. 2017, 50, 423002.

(5) Coropceanu, V.; Cornil, J.; da Silva Filho, D. A.; Olivier, Y.; Silbey, R.; Brédas, J. L. Charge transport in organic semiconductors. Chem. Rev. 2007, 107, 926-952.

(6) Hinderhofer, A.; Schreiber, F. Organic-Organic Heterostructures: Concepts and Applications. ChemPhysChem 2013, 13, 628-643.

(7) Jones, A. O. F.; Rothel, C.; Lassnig, R.; Bedoya-Martinez, O. N.; Christian, P.; Salzmann, I.; Kunert, B.; Winkler, A.; Resel, R. Solution of an elusive pigment crystal structure from a thin film: a combined X-ray diffraction and computational study. CrystEngComm 2017, 19, 1902-1911.

(8) Chen, W.; Qi, D. C.; Huang, H.; Gao, X. Y.; Wee, A. T. S. Organic-Organic Heterojunction Interfaces: Effect of Molecular Orientation. Adv. Funct. Mater. 2011, 21, 410-424.

(9) Ueno, N.; Kera, S. Electron spectroscopy of functional organic thin films: Deep insights into valence electronic structure in relation to charge transport property. Prog. Surf. Sci. 2008, 83, 490-557. 
(10) Kim, V. O.; Broch, K.; Belova, V.; Chen, Y. S.; Gerlach, A.; Schreiber, F.; Tamura, H.; Della Valle, R. G.; D’Avino, G.; Salzmann, I.; Beljonne, D.; Rao, A.; Friend, R. Singlet exciton fission via an intermolecular charge transfer state in coevaporated pentacene-perfluoropentacene thin films. J. Chem. Phys. 2019, 151, 164706.

(11) Chen, H.-Y.; Chao, I. Effect of perfluorination on the charge-transport properties of organic semiconductors: density functional theory study of perfluorinated pentacene and sexithiophene. Chem. Phys. Lett. 2005, 401, $539-545$.

(12) Ruiz Delgado, M. C.; Pigg, K. R.; da Silva Filho, D. A.; Gruhn, N. E.; Sakamoto, Y.; Suzuki, T.; Osuna, R. M.; Casado, J.; Hernández, V.; Navarrete, J. T. L.; Martinelli, N. G.; Cornil, J.; Sánchez-Carrera, R. S.; Coropceanu, V.; Brédas, J.-L. Impact of Perfluorination on the Charge-Transport Parameters of Oligoacene Crystals. J. Am. Chem. Soc. 2009, 131, 1502-1512.

(13) Ryno, S. M.; Lee, S. R.; Sears, J. S.; Risko, C.; Brédas, J.-L. Electronic Polarization Effects upon Charge Injection in Oligoacene Molecular Crystals: Description via a Polarizable Force Field. J. Phys. Chem. C 2013, 117, 13853-13860.

(14) D’Avino, G.; Muccioli, L.; Castet, F.; Poelking, C.; Andrienko, D.; Soos, Z. G.; Cornil, J.; Beljonne, D. Electrostatic phenomena in organic semiconductors: fundamentals and implications for photovoltaics. J. Phys.: Condens. Matter 2016, 28, 433002.

(15) Schwarze, M.; Tress, W.; Beyer, B.; Gao, F.; Scholz, R.; Poelking, C.; Ortstein, K.; Gunther, A. A.; Kasemann, D.; Andrienko, D.; Leo, K. Band structure engineering in organic semiconductors. Science 2016, 352, 1446-1449.

(16) Li, J.; D'Avino, G.; Duchemin, I.; Beljonne, D.; Blase, X. Accurate description of charged excitations in molecular solids from embedded many-body perturbation theory. Phys. Rev. B 2018, 97, 035108. 
(17) Zojer, E.; Taucher, T. C.; Hofmann, O. T. The Impact of Dipolar Layers on the Electronic Properties of Organic/Inorganic Hybrid Interfaces. Adv. Mater. Interfaces 2019, 6, 1900581-32.

(18) Salzmann, I.; Duhm, S.; Heimel, G.; Oehzelt, M.; Kniprath, R.; Johnson, R. L.; Rabe, J. P.; Koch, N. Tuning the Ionization Energy of Organic Semiconductor Films: The Role of Intramolecular Polar Bonds. J. Am. Chem. Soc. 2008, 130, 12870-12871.

(19) Duhm, S.; Heimel, G.; Salzmann, I.; Glowatzki, H.; Johnson, R. L.; Vollmer, A.; Rabe, J. P.; Koch, N. Orientation-dependent ionization energies and interface dipoles in ordered molecular assemblies. Nat. Mater. 2008, 7, 326.

(20) Yoshida, H.; Yamada, K.; Tsutsumi, J.; Sato, N. Complete description of ionization energy and electron affinity in organic solids: Determining contributions from electronic polarization, energy band dispersion, and molecular orientation. Phys. Rev. $B$ 2015, 92, 075145.

(21) Chen, W.; Huang, H.; Chen, S.; Huang, Y. L.; Gao, X. Y.; Wee, A. T. S. Molecular Orientation-Dependent Ionization Potential of Organic Thin Films. Chem. Mater. 2008, 20, 7017-7021.

(22) Heimel, G.; Salzmann, I.; Duhm, S.; Rabe, J. P.; Koch, N. Intrinsic Surface Dipoles Control the Energy Levels of Conjugated Polymers. Adv. Funct. Mater. 2009, 19, 38743879 .

(23) Fukagawa, H.; Yamane, H.; Kataoka, T.; Kera, S.; Nakamura, M.; Kudo, K.; Ueno, N. Origin of the highest occupied band position in pentacene films from ultraviolet photoelectron spectroscopy: Hole stabilization versus band dispersion. Phys. Rev. B 2006, $73,245310$.

(24) Duhm, S.; Salzmann, I.; Heimel, G.; Oehzelt, M.; Haase, A.; Johnson, R. L.; Rabe, J. P.; 
Koch, N. Controlling energy level offsets in organic/organic heterostructures using intramolecular polar bonds. Appl. Phys. Lett. 2009, 94, 033304.

(25) Salzmann, I. et al. Epitaxial Growth of $\pi$-Stacked Perfluoropentacene on GrapheneCoated Quartz. ACS Nano 2012, 6, 10874-10883.

(26) Götzen, J.; Käfer, D.; Wöll, C.; Witte, G. Growth and structure of pentacene films on graphite: Weak adhesion as a key for epitaxial film growth. Phys. Rev. B 2010, 81, 085440 .

(27) Koch, N.; Vollmer, A.; Salzmann, I.; Nickel, B.; Weiss, H.; Rabe, J. P. Evidence for temperature-dependent electron band dispersion in pentacene. Phy. Rev. Lett. 2006, $96,156803$.

(28) Hinderhofer, A.; Frank, C.; Hosokai, T.; Resta, A.; Gerlach, A.; Schreiber, F. Structure and morphology of coevaporated pentacene-perfluoropentacene thin films. J. Chem. Phys. 2011, 134, 104702.

(29) Félix, R.; Volz, K.; Gries, K. I. Codeposited pentacene:perfluoropentacene grown on SiO2: A microstructural study by transmission electron microscopy. J. Cryst. Growth 2017, $458,87-95$.

(30) Broch, K.; Heinemeyer, U.; Hinderhofer, A.; Anger, F.; Scholz, R.; Gerlach, A.; Schreiber, F. Optical evidence for intermolecular coupling in mixed films of pentacene and perfluoropentacene. Phys. Rev. B 2011, 83, 245307.

(31) Rinn, A.; Breuer, T.; Wiegand, J.; Beck, M.; Hübner, J.; Döring, R. C.; Oestreich, M.; Heimbrodt, W.; Witte, G.; Chatterjee, S. Interfacial Molecular Packing Determines Exciton Dynamics in Molecular Heterostructures: The Case of Pentacene-Perfluoropentacene. ACS Appl. Mater. Interfaces 2017, 9, 42020-42028. 
(32) Salzmann, I.; Duhm, S.; Heimel, G.; Rabe, J. P.; Koch, N.; Oehzelt, M.; Sakamoto, Y.; Suzuki, T. Structural Order in Perfluoropentacene Thin Films and Heterostructures with Pentacene. Langmuir 2008, 24, 7294-7298.

(33) Breuer, T.; Witte, G. Thermally activated intermixture in pentaceneperfluoropentacene heterostructures. J. Chem. Phys. 2013, 138, 114901.

(34) Schwarze, M. et al. Impact of molecular quadrupole moments on the energy levels at organic heterojunctions. Nat. Commun. 2019, 10, 2466.

(35) Topham, B. J.; Soos, Z. G. Ionization in organic thin films: Electrostatic potential, electronic polarization, and dopants in pentacene films. Phys. Rev. B 2011, 84, 165405.

(36) Duhm, S.; Xin, Q.; Hosoumi, S.; Fukagawa, H.; Sato, K.; Ueno, N.; Kera, S. Charge Reorganization Energy and Small Polaron Binding Energy of Rubrene Thin Films by Ultraviolet Photoelectron Spectroscopy. Adv. Mater. 2012, 24, 901-905.

(37) Moser, A. Crystal Structure Solution Based on Grazing Incidence X-ray Diffraction: Software Development and Application to Organic Films. Thesis, Graz University of Technology, 2012.

(38) Besler, B. H.; Merz, K. M.; Kollman, P. A. Atomic charges derived from semiempirical methods. J. Comput. Chem. 1990, 11, 431-439.

(39) Frisch, M. J. et al. Gaussian09 Revision D.01. Gaussian Inc. Wallingford CT 2009.

(40) Holmes, D.; Kumaraswamy, S.; Matzger, A. J.; Vollhardt, K. P. C. On the Nature of Nonplanarity in the [N]Phenylenes. Chem. Eur. J. 1999, 5, 3399-3412.

(41) Siegrist, T.; Besnard, C.; Haas, S.; Schiltz, M.; Pattison, P.; Chernyshov, D.; Batlogg, B.; Kloc, C. A Polymorph Lost and Found: The High-Temperature Crystal Structure of Pentacene. Adv. Mater. 2007, 19, 2079-2082. 
(42) Sakamoto, Y.; Suzuki, T.; Kobayashi, M.; Gao, Y.; Fukai, Y.; Inoue, Y.; Sato, F.; Tokito, S. Perfluoropentacene: High-Performance p-n Junctions and Complementary Circuits with Pentacene. J. Am. Chem. Soc. 2004, 126, 8138-8140.

(43) Jorgensen, W. L.; Maxwell, D. S.; Tirado-Rives, J. Development and Testing of the OPLS All-Atom Force Field on Conformational Energetics and Properties of Organic Liquids. J. Am. Chem. Soc. 1996, 118, 11225-11236.

(44) Watkins, E. K.; Jorgensen, W. L. Perfluoroalkanes: Conformational Analysis and Liquid-State Properties from ab Initio and Monte Carlo Calculations. J. Phys. Chem. A 2001, 105, 4118-4125.

(45) Adamo, C.; Barone, V. Toward reliable density functional methods without adjustable parameters: The PBE0 model. J. Chem. Phys. 1999, 110, 6158-6170.

(46) Dovesi, R.; Orlando, R.; Erba, A.; Zicovich-Wilson, C. M.; Civalleri, B.; Casassa, S.; Maschio, L.; Ferrabone, M.; De La Pierre, M.; D’Arco, P.; Noël, Y.; Causà, M.; Rérat, M.; Kirtman, B. CRYSTAL14: A program for the ab initio investigation of crystalline solids. Int. J. Quantum Chem. 2014, 114, 1287-1317.

(47) Valeev, E. F.; Coropceanu, V.; da Silva Filho, D. A.; Salman, S.; Brédas, J.-L. Effect of Electronic Polarization on Charge-Transport Parameters in Molecular Organic Semiconductors. J. Am. Chem. Soc. 2006, 128, 9882-9886.

(48) Neese, F. The ORCA program system. WIREs Comput. Mol. Sci. 2012, 2, 73-78.

(49) Coropceanu, V.; Malagoli, M.; da Silva Filho, D. A.; Gruhn, N. E.; Bill, T. G.; Brédas, J. L. Hole- and Electron-Vibrational Couplings in Oligoacene Crystals: Intramolecular Contributions. Phys. Rev. Lett. 2002, 89, 275503.

(50) Kera, S.; Ueno, N. Photoelectron spectroscopy on the charge reorganization energy 
and small polaron binding energy of molecular film. J. Electron Spectrosc. Relat. Phenom. 2015, 204, 2-11.

(51) Tsiper, E. V.; Soos, Z. G. Charge redistribution and polarization energy of organic molecular crystals. Phys. Rev. B 2001, 64, 195124.

(52) Soos, Z.; Tsiper, E. V.; Pascal, R. Charge redistribution and electronic polarization in organic molecular crystals. Chem. Phys. Lett. 2001, 342, 652 - 658.

(53) D’Avino, G.; Muccioli, L.; Zannoni, C.; Beljonne, D.; Soos, Z. G. Electronic Polarization in Organic Crystals: A Comparative Study of Induced Dipoles and Intramolecular Charge Redistribution Schemes. J. Chem. Theory Comput. 2014, 10, 4959-4971.

(54) Siegrist, T.; Kloc, C.; Schön, J. H.; Batlogg, B.; Haddon, R. C.; Berg, S.; Thomas, G. A. Enhanced Physical Properties in a Pentacene Polymorph. Angew. Chem. Int. Ed. 2001, 40, 1732-1736.

(55) Simbrunner, J.; Simbrunner, C.; Schrode, B.; Rothel, C.; Bedoya-Martinez, N.; Salzmann, I.; Resel, R. Indexing of grazing-incidence X-ray diffraction patterns: the case of fibre-textured thin films. Acta Cryst. A 2018, 74, 373-387.

(56) Simbrunner, J.; Hofer, S.; Schrode, B.; Garmshausen, Y.; Hecht, S.; Resel, R.; Salzmann, I. Indexing grazing-incidence $X$-ray diffraction patterns of thin films: lattices of higher symmetry. J. Appl. Crystallogr. 2019, 52, 428-439.

(57) Schiefer, S.; Huth, M.; Dobrinevski, A.; Nickel, B. Determination of the Crystal Structure of Substrate-Induced Pentacene Polymorphs in Fiber Structured Thin Films. J. Am. Chem. Soc. 2007, 129, 10316-10317.

(58) Nabok, D.; Puschnig, P.; Ambrosch-Draxl, C.; Werzer, O.; Resel, R.; Smilgies, D. M. Crystal and electronic structures of pentacene thin films from grazing-incidence $x-$ ray diffraction and first-principles calculations. Phys. Rev. B 2007, 76, 235322. 
(59) Mattheus, C. C.; Dros, A. B.; Baas, J.; Meetsma, A.; de Boer, J. L.; Palstra, T. T. Polymorphism in pentacene. Acta Crystal. C 2001, 57, 939-941.

(60) Mattheus, C. C.; Dros, A. B.; Baas, J.; Oostergetel, G. T.; Meetsma, A.; de Boer, J. L.; Palstra, T. T. M. Identification of polymorphs of pentacene. Synth. Met. 2003, 138, 475-481.

(61) Félix, R.; Breuer, T.; Witte, G.; Volz, K.; Gries, K. I. Microstructural study of codeposited pentacene:perfluoropentacene grown on $\mathrm{KCl}$ by TEM techniques. J. Cryst. Growth 2017, 471, $29-36$.

(62) Salzmann, I.; Nabok, D.; Oehzelt, M.; Duhm, S.; Moser, A.; Heimel, G.; Puschnig, P.; Ambrosch-Draxl, C.; Rabe, J. P.; Koch, N. Structure Solution of the 6,13-Pentacenequinone Surface-Induced Polymorph by Combining X-ray Diffraction Reciprocal-Space Mapping and Theoretical Structure Modeling. Cryst. Growth Des. 2011, 11, 600-606.

(63) Pichler, A.; Resel, R.; Neuhold, A.; Dingemans, T.; Schwabegger, G.; Moret, M.; Simbrunner, C.; Salzmann, I. Crystal structure determination of organic thin-films: the example of 2,2':6',2"-ternaphthalene. Z. Kristallogr. 2014, 229, 385-393.

(64) Truger, M.; Roscioni, O. M.; Röthel, C.; Kriegner, D.; Simbrunner, C.; Ahmed, R.; Głowacki, E. D.; Simbrunner, J.; Salzmann, I.; Coclite, A. M.; Jones, A. O. F.; Resel, R. Surface-Induced Phase of Tyrian Purple (6,6'-Dibromoindigo): Thin Film Formation and Stability. Cryst. Growth Des. 2016, 16, 3647-3655.

(65) Brock, C. P.; Dunitz, J. D. Towards a Grammar of Crystal Packing. Chem. Mater. 1994, $6,1118-1127$.

(66) Nakayama, Y.; Tsuruta, R.; Moriya, N.; Hikasa, M.; Meissner, M.; Yamaguchi, T.; Mizuno, Y.; Suzuki, T.; Koganezawa, T.; Hosokai, T.; Ueba, T.; Kera, S. Widely Dis- 
persed Intermolecular Valence Bands of Epitaxially Grown Perfluoropentacene on Pentacene Single Crystals. J. Phys. Chem. Lett. 2019, 10, 1312-1318.

(67) Berkebile, S.; Puschnig, P.; Koller, G.; Oehzelt, M.; Netzer, F. P.; Ambrosch-Draxl, C.; Ramsey, M. G. Electronic band structure of pentacene: An experimental and theoretical study. Phys. Rev. B 2008, 77, 115312.

(68) Kera, S.; Fukagawa, H.; Kataoka, T.; Hosoumi, S.; Yamane, H.; Ueno, N. Spectroscopic evidence of strong pi-pi interorbital interaction in a lead-phthalocyanine bilayer film attributed to the dimer nanostructure. Phys. Rev. B 2007, 75, 121305(R).

(69) Kashimoto, Y.; Yonezawa, K.; Meissner, M.; Gruenewald, M.; Ueba, T.; Kera, S.; Forker, R.; Fritz, T.; Yoshida, H. The Evolution of Intermolecular Energy Bands of Occupied and Unoccupied Molecular States in Organic Thin Films. J. Phys. Chem. C 2018, 122, 12090-12097.

(70) Zhu, L.; Yi, Y.; Li, Y.; Kim, E.-G.; Coropceanu, V.; Brédas, J.-L. Prediction of Remarkable Ambipolar Charge-Transport Characteristics in Organic Mixed-Stack ChargeTransfer Crystals. J. Am. Chem. Soc. 2012, 134, 2340-2347.

(71) Ji, R.-R.; Wang, Q.; Hu, J.-X.; Duhm, S. Impact of room temperature on pentacene thin film growth and electronic structure. Can. J. Chem. 2017, 95, 1130-1134.

(72) Kera, S.; Hosoumi, S.; Sato, K.; Fukagawa, H.; Nagamatsu, S.-i.; Sakamoto, Y.; Suzuki, T.; Huang, H.; Chen, W.; Wee, A. T. S.; Coropceanu, V.; Ueno, N. Experimental Reorganization Energies of Pentacene and Perfluoropentacene: Effects of Perfluorination. J. Phys. Chem. C 2013, 117, 22428-22437.

(73) Koch, N.; Vollmer, A.; Duhm, S.; Sakamoto, Y.; Suzuki, T. The effect of fluorination on pentacene/gold interface energetics and charge reorganization energy. Adv. Mater. 2007, 19, 112-116. 
(74) Venables, J. A.; Spiller, G. D. T.; Hanbucken, M. Nucleation and Growth of ThinFilms. Rep. Prog. Phys. 1984, 47, 399-459.

(75) Kowarik, S. Thin film growth studies using time-resolved x-ray scattering. J. Phys.: Condens. Matter 2017, 29, 043003-19.

(76) Yang, J.; Yan, D.; Jones, T. S. Molecular Template Growth and Its Applications in Organic Electronics and Optoelectronics. Chem. Rev. 2015, 115, 5570-5603.

(77) Shionoiri, M.; Kozasa, M.; Kera, S.; Okudaira, K. K.; Ueno, N. Preparation Conditions of Pentacene Monolayer on Graphite Surface Studied by Photoemission Electron Microscopy. Jpn. J. Appl. Phys. 2007, 46, 1625-1629.

(78) Tsiper, E. V.; Soos, Z. G. Electronic polarization in pentacene crystals and thin films. Phys. Rev. B 2003, 68, 085301.

(79) Seah, M. P.; Dench, W. A. Quantitative electron spectroscopy of surfaces: A standard data base for electron inelastic mean free paths in solids. Surf. Interface Anal. 1979, 1, $2-11$.

(80) Clark, P. A.; Brogli, F.; Heilbronner, E. The $\pi$-Orbital Energies of the Acenes. Helv. Chim. Acta 1972, 55, 1415-1428. 


\section{Graphical TOC Entry}

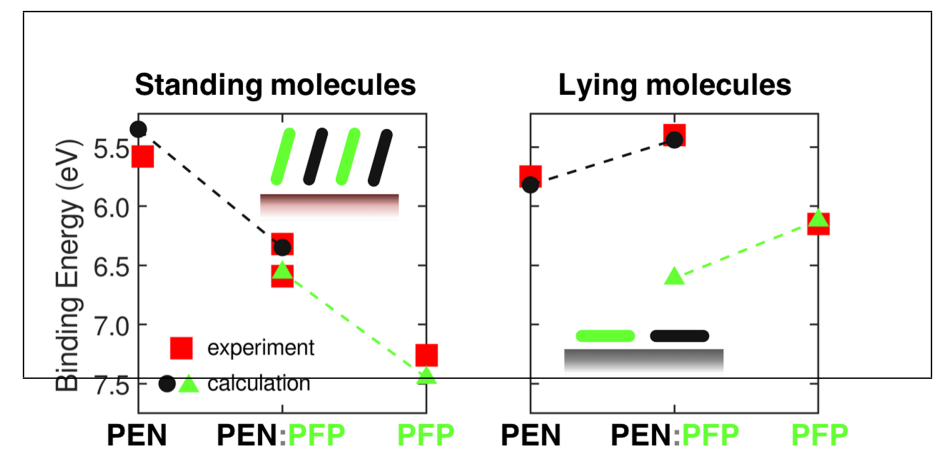

